\title{
Crecimiento secundario inusual en raíz principal de Pfaffia gnaphalioides (L. Fil.) Mart. (Amaranthaceae)
}

\author{
Mónica Alicia Grosso ${ }^{1}$
}

Recibido: 20/10/2005. Aceptado: 27/07/2006

\begin{abstract}
RESUMEN - (Crecimiento secundario inusual en raíz principal de Pfaffia gnaphalioides (L. Fil.) Mart. (Amaranthaceae)). Raíces de Pfaffia se comercializan como "ginseng brasilero" y extractos crudos de raíces de Pfaffia glomerata tienen actividad analgésica y desinflamatoria. Pfaffia gnaphalioides es herbácea perenne y crece en el área serrana del sur de la Provincia de Córdoba, Argentina. El objetivo de este trabajo es estudiar el crecimiento secundario inusual en raíz principal de Pfaffia gnaphalioides. Se estudió en invernáculo la forma de crecimiento durante los dos primeros años relacionando la parte aérea y subterránea. Se realizaron calicatas in situ para analizar el sistema radical. La raíz principal desarrolla raíces laterales, plagiótropas, con yemas que forman brotes aéreos de innovación. En cortes transversales de raíz principal se observa desarrollo secundario inusual. Un meristema lateral produce corteza secundaria hacia afuera y tejido conjuntivo y una sucesión de cambia vascular hacia adentro. Cuando cada cambium vascular es producido, el meristema lateral que se encuentra hacia afuera de éste tiende a permanecer quiescente, retornando a la actividad cuando el cambium vascular interno a éste permanece menos activo. Los cordones vasculares secundarios están dispuestos en anillos concéntricos sin radios parenquimáticos. La presencia de abundante parénquima conteniendo almidón en la estructura inusual de raíz en Pfaffia gnaphalioides, constituye una buena adaptación a condiciones xéricas.
\end{abstract}

Palabras claves: Pfaffia gnaphalioides, Amaranthaceae, raíces gemíferas, cambia adicionales, lateral meristem

\begin{abstract}
Secondary and unusual growth in main root of Pfaffia gnaphalioides (L. Fil.) Mart. (Amaranthaceae)). Pfaffia roots have been commercialized as "brazilian ginseng" and crude extracts of Pfaffia glomerata roots have analgesic and disinflamatory activity. Pfaffia gnaphalioides is a perennial herb and grows in southern Córdoba, Argentina. The objective of this work is to analyze root systems in Pfaffia gnaphalioides. Growth of above and belowground organs was observed for two years in a greenhouse. Belowground organs were analyzed in situ in hillcountry shrublands, by digging soil pits. The root system is characterized by presence of a main root and superficial plagiotropic lateral roots. Lateral roots show renewal buds that give rise to new aerial innovation shoots. Unusual secondary growth is observed in the main root. A lateral meristem produces secondary cortex to the outside and conjuntive tissue and a succesion of vascular cambia to the inside. As each vascular cambium is produced, the lateral meristem outside it tends to become quiescent, returning to activity when the vascular cambium internal to it has become less active. Secondary vascular strands are arranged in concentric rings without rays. The presence of abundant parenchyma containing starch in unusual structure of Pfaffia gnaphalioides, constitutes an adaptation to xeric conditions.
\end{abstract}

Key words: Pfaffia gnaphalioides, Amaranthaceae, root-borne shoots, successive cambia, lateral meristem

\section{Introducción}

Pfaffia gnaphalioides (L. Fil.) Mart. es una especie herbácea perenne con una amplia distribución: Brasil, Bolivia, Paraguay, Uruguay y, en Argentina, en las provincias de Buenos Aires, Catamarca, Córdoba, Entre Ríos, Jujuy, La Pampa, Salta, Santiago del Estero, Santa Fe, San Luis y Tucumán. Es conocida su importancia económica debido a que las raíces de especies de Pfaffia L. han sido comercializadas como "ginseng brasilero" a pesar de que no están claras las especificaciones farmacológicas (Vigo et al. 2004). Asimismo, extractos crudos de raíces de Pfaffia glomerata (Spreng) Pedersen son utilizados con actividad analgésica y desinflamatoria (Neto et al. 2005). Cabe destacar también que a partir de la inducción de la formación de callos de tallo y raíz de esta misma especie se producen saponinas (Kagiki et al. 2004).

La arquitectura de la raíz es un aspecto fundamental en el desarrollo de la planta teniendo en cuenta la necesidad de explotar un ambiente espacialmente heterogéneo. Muchos recursos del suelo están desigualmente distribuidos o están sujetos a una reducción localizada, por eso es que el desarrollo espacial del sistema radical será en gran medida

\footnotetext{
1 Universidad Nacional de Río Cuarto, Facultad de Agronomía y Veterinaria, Morfología Vegetal, 5800 Río Cuarto Córdoba, Argentina (mgrosso@ayv.unrc.edu.ar)
} 
determinado por la habilidad de una planta de explotar estos recursos (Lynch 1995). El patrón de crecimiento del sistema radical de una especie está determinado principalmente por su caracterización genética, como fue corroborado por Kutschera \& Lichtenegger (1992). Sin embargo, también está influenciado por la disponibilidad de agua, la temperatura, los nutrientes y las condiciones químicas y biológicas de los distintos horizontes del suelo (Fahn 1985; Schubert 1991).

A nivel anatómico se han realizado numerosos estudios en Amaranthaceae, Chenopodiaceae y Nyctaginaceae, demostrando que el crecimiento secundario en el tallo es inusual (Metcalfe \& Chalk 1957; 1983; Philipson \& Ward 1965; Balfour 1965; Rajput \& Rao 1999; Rajput 2001; 2002), y lo mismo ocurre en raíces de Nyctaginaceae (Carlquist 2004). En el tallo de Chenopodium ambrosioides L. (Chenopodiaceae), Gattuso \& Gattuso (1985) observaron que los sucesivos cambia bidireccionales se originan del parénquima floemático periférico, produciendo centrípetamente elementos de xilema y parénquima lignificado $y$, centrífugamente, elementos de floema y parénquima conjuntivo. Rajput \& Rao (1999) sostienen que el tallo de Pupalia lappacea (L.) Juss (Amaranthaceae) está compuesto por cinco a seis anillos sucesivos de cambia. Cada anillo de cambium se desarrolla a partir del parénquima floemático producido por el cambium precedente. Éste está formado por un sector fascicular que desarrolla elementos de xilema y floema, y un sector interfascicular que da origen centrípetamente a fibras xilemáticas y centrífugamente a parénquima axial. La estructura resultante carece de radios parenquimáticos. Rajput \& Rao (1998) también observan, en especies de Boerhaavia L. (Nyctaginaceae), la presencia de crecimiento secundario anómalo en tallos caracterizado por la presencia de anillos sucesivos de cambium que originan cordones vasculares inmersos en un parénquima sin diferenciación de radios parenquimáticos.

En relación al almacenamiento de sustancias, las raíces reservantes son consideradas estrategias evolutivas eficientes y, en general, se asocia este carácter con estructuras inusuales (Pate 1993; Esau 1985). La presencia de carbohidratos en raíces puede estar también relacionada con la tolerancia al frío permitiendo la sobrevivencia en el invierno cuando muere la parte aérea y el consiguiente rebrote en primavera (Baur Höch et al. 1990; Rosnes et al. 1993). Canny (1998) sostiene que la presencia de almidón en el parénquima podría favorecer la absorción de agua por parte de la raíz. Cuando hay disponibilidad de agua en el suelo, por hidrólisis del almidón en carbohidratos solubles, se produce un aumento del potencial osmótico celular, por lo cual aumenta la tendencia a retener agua por parte de los tejidos. Asimismo, dentro del cilindro vascular se genera una presión tal que favorece un eficiente flujo de agua.

El objetivo de este trabajo es analizar el desarrollo del crecimiento secundario inusual en raíz principal de Pfaffia gnaphalioides (L. Fil.) Mart. en el sur de la provincia de Córdoba, Argentina.

\section{Material y métodos}

Pfaffia gnaphalioides (L. Fil.) Mart. se encuentra en el sur de la provincia de Córdoba, Argentina. El estudio se llevó a cabo a los $33^{\circ} \mathrm{S}$ y $65^{\circ} \mathrm{O}$. El tipo fisonómico es Arbustal, llamado vulgarmente "romerillal" porque la especie dominante es Heterothalamus alienus (Spreng.) O.K. "romerillo". Este tipo de vegetación se manifiesta a partir de los 800 m.s.n.m. y se localiza en lomas y laderas muy ventosas y secas con suelo poco profundo o incipiente. Desde el punto de vista climático, el área se caracteriza por presentar inviernos secos, con algunas precipitaciones en forma de nieve, veranos lluviosos, vientos frecuentes y gran amplitud térmica.

Se recolectaron semillas de Pfaffia gnaphalioides, las que fueron sembradas en invernáculo. En estas plantas se estudió el sistema radical de la especie durante los dos primeros años. Paralelamente, se realizaron calicatas en su ambiente para analizar la disposición espacial del sistema radical. Para ésto, se descubrieron progresivamente cada una de las partes y, con ayuda de agujas, pinzas y pinceles, se siguió el crecimiento de la raíz principal y del resto de los órdenes. De esta manera, se observaron longitud, diámetro, ángulo de inserción y dirección de crecimiento de las raíces (Kutschera-Mitter 1960).

Las muestras de raíz principal de una planta obtenida en el laboratorio fueron fijadas en FAA (formaldehído, ácido acético, etanol 50\%,1:1:18 v/v) (Johansen 1940), luego se deshidrataron en una serie de alcohol etílico, se clarificaron en xilol y se incluyeron en Histowax. Se realizaron cortes secuenciales transversales con un ultramicrótomo $(7 \mu \mathrm{m})$ y se colorearon con Safranina-Verde rápido (Johansen 1940). Para detectar la presencia de almidón se utilizó el reactivo de Lugol al 1\% como técnica histoquímica (Johansen 1940). Las fotografías fueron obtenidas con un microscopio Axiophot-Zeiss. 


\section{Resultados y discusión}

El sistema radical en Pfaffia gnaphalioides (L. Fil.) Mart. es alorrizo, siendo la relación parte subterránea-parte aérea uno a uno en una planta de 60 días, con una longitud de $8 \mathrm{~cm}$ y un diámetro de $0,2 \mathrm{~cm}$ (Fig. 1). En su segundo año de crecimiento, la raíz principal tiene desarrollo ortótropo, alcanza $0,8 \mathrm{~cm}$ de diámetro y $50 \mathrm{~cm}$ de longitud aproximadamente. A los $5 \mathrm{~cm}$ de la base de la raíz principal se originan varias raíces laterales plagiótropas, con un diámetro de $0,4 \mathrm{~cm}$ y longitud de hasta $40 \mathrm{~cm}$. Estas raíces presentan yemas que se localizan cada $10 \mathrm{~cm}$ aproximadamente. Estas yemas pueden desarrollar brotes de innovación durante el mismo año o pueden quedar latentes durante el período desfavorable para originar nuevos brotes al año siguiente (primaveraverano), repitiendo el esquema arquitectural de la planta madre (Tab. 1, Fig. 2, 3). Por lo tanto, a partir del segundo año, la propagación vegetativa se realiza
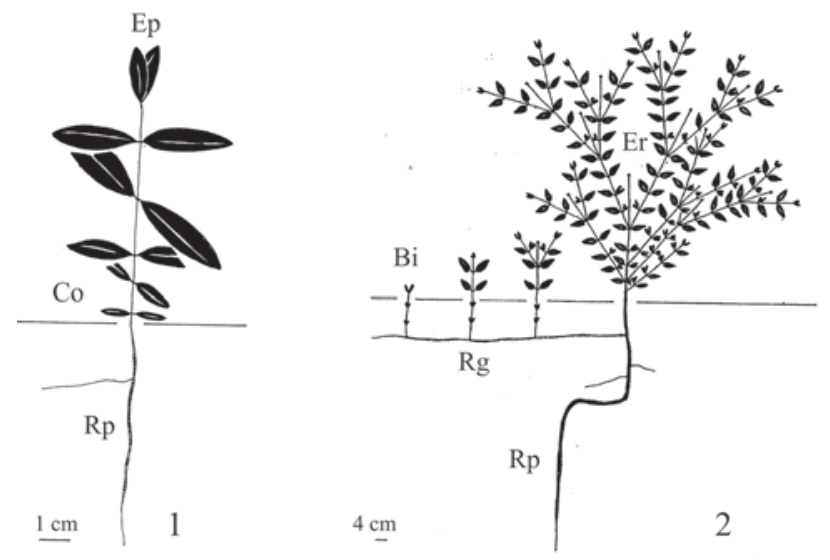

Figuras 1-2. Pfaffia gnaphalioides (L. Fil.) Mart. 1. Desarrollo monopodial en planta joven de 60 días obtenida en condiciones de laboratorio. 2. Desarrollo simpodial y formación de raíz gemífera en una planta de dos años obtenida en condiciones de laboratorio. $\mathrm{Bi}=$ brote de innovación $; \mathrm{Co}=$ cotiledones; $\mathrm{Ep}=$ eje primario; $\mathrm{Rg}=$ raíz gemífera; $\mathrm{Rp}=$ raíz principal.

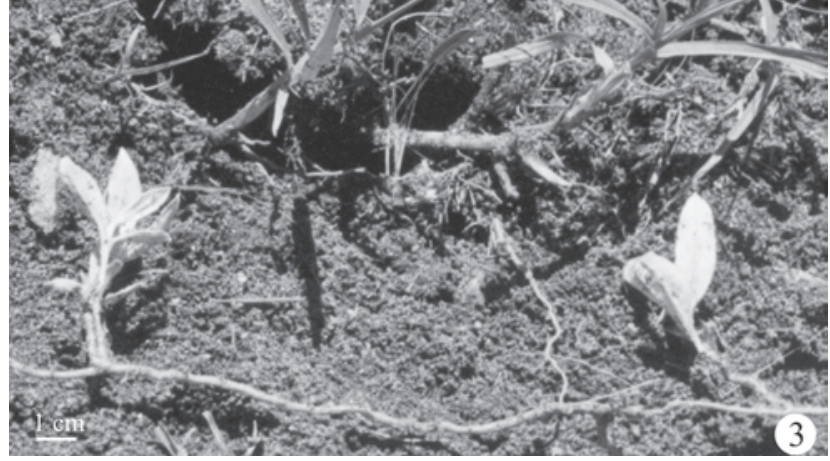

Figura 3. Raíz gemífera en Pfaffia gnaphalioides (L. Fil.) Mart. Campo experimental "Las Guindas" (área serrana). Universidad Nacional de Río Cuarto. Córdoba.

a partir de la formación de nuevos brotes de innovación que se desarrollan a partir de raíces gemíferas, lo cual permite una extensa colonización, cubriendo rápidamente el área en forma de manchones característicos.

Los sistemas radicales están determinados principalmente por un componente genético según Kutschera \& Lichtenegger (1992), sin embargo las condiciones ambientales también influyen en su desarrollo (Schubert 1991). Fahn (1985) estudió las adaptaciones de las raíces a condiciones xéricas y observó que la presencia de raíces con desarrollo horizontal es uno de los caracteres adaptativos de las mismas a dichas condiciones. La presencia de raíces gemíferas plagiótropas en Paffia gnaphalioides constituye una forma de protección para esta especie en períodos de sequía o baja temperatura, ya que garantizan el rebrote en épocas favorables. Ésto estaría de acuerdo con lo expresado por Lynch (1995), quien sostiene que la arquitectura del sistema radical presenta una configuración espacial con significado funcional.

En los cortes secuenciales de la raíz principal se observa un desarrollo secundario inusual con la presencia de sucesivos cambia adicionales de

Tabla 1. Plantas de Pfaffia gnaphalioides (L. Fil.) Mart. obtenidas en condiciones de laboratorio. Longitud y diámetro de raíz principal. Raíz lateral gemífera, orientación, diámetro y ubicación de yemas de renuevo.

\begin{tabular}{|c|c|c|c|}
\hline Tipo de raíz & Características & Planta de 60 días $(\mathrm{n}=10)$ & Planta de 2 años $(\mathrm{n}=10)$ \\
\hline \multirow[t]{2}{*}{ Raíz principal } & Longitud (cm) & 8 & 50 \\
\hline & Diámetro (cm) & 0,2 & 0,8 \\
\hline \multirow[t]{3}{*}{ Raíz lateral gemífera } & Diámetro (cm) & - & 0,4 \\
\hline & Longitud (cm) & - & 40 \\
\hline & Yemas de renuevo & - & cada $10 \mathrm{~cm}$ aprox. \\
\hline
\end{tabular}


actividad limitada originados a partir del meristema lateral. A los 0,5 cm del ápice (Fig. 4) se observa una raíz con crecimiento secundario, donde el meristema lateral da origen a la corteza secundaria hacia afuera y sucesivos cambia y tejidos conjuntivos hacia el interior. Cuando cada cambium vascular adicional es producido, el meristema lateral que se encuentra hacia afuera de éste permanece latente, retornando a la actividad cuando el cambium vascular interno a éste permanece menos activo. El primer cambium tiene actividad normal, originando xilema con escasa proporción de parénquima y floema bien desarrollado. A partir del meristema lateral comienza el desarrollo del primer anillo de cambium adicional formado por dos hileras de células cambiales que dan origen a cordones vasculares inmersos en parénquima. La aparición de la estructura secundaria ocurre de manera no sincronizada y el cambium diferencia primero xilema y luego floema. A los $1,5 \mathrm{~cm}$ del ápice radical (Fig. 5) el primer cambium adicional se observa formado por tres hileras de células y se han agregado primero elementos xilemáticos y luego floemáticos en aproximadamente seis cordones vasculares de mayor tamaño que los que se forman en los sucesivos anillos cambiales siguientes, pero predomina el tejido parenquimático. A los $3,5 \mathrm{~cm}$ del ápice, el primer
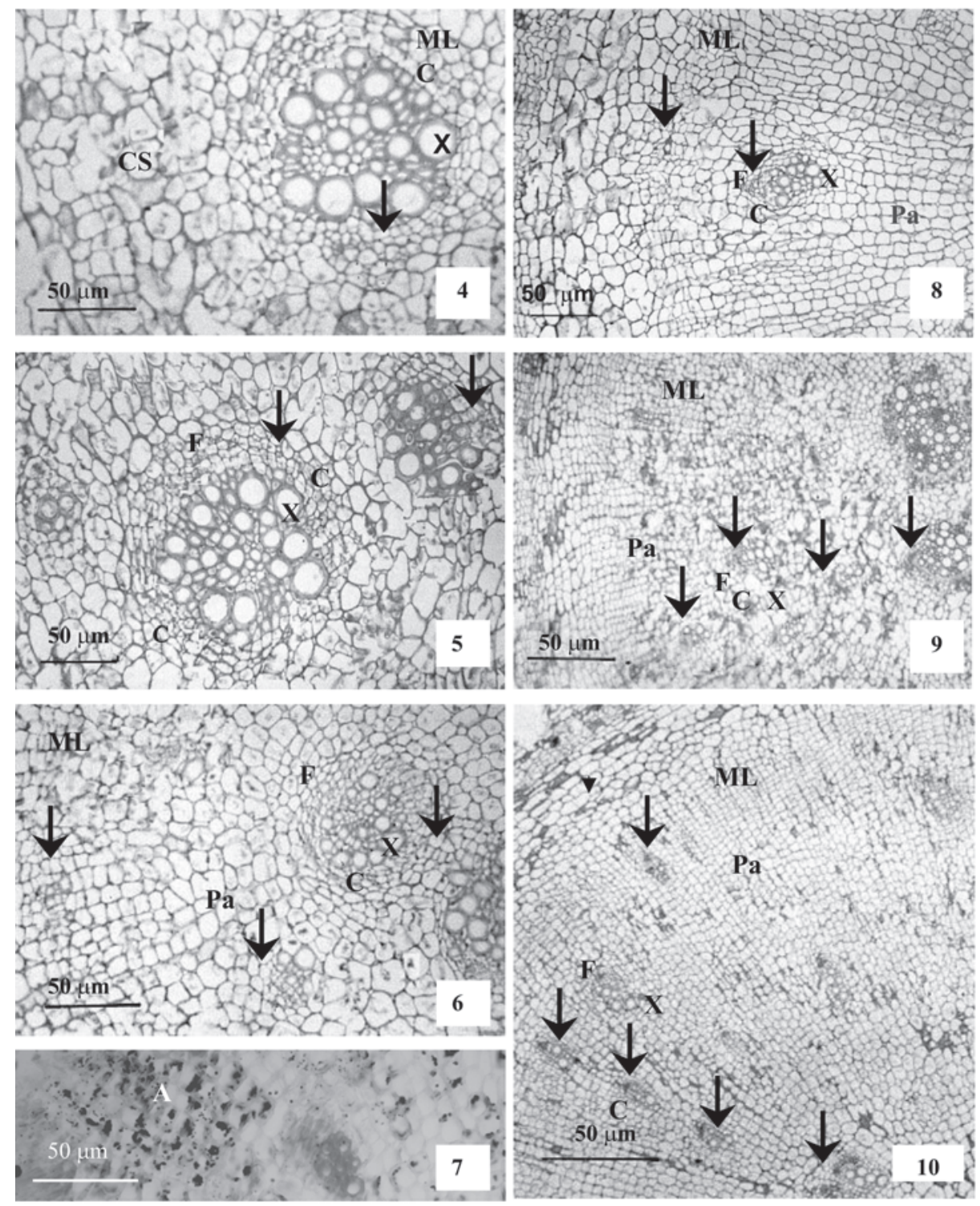

Figuras 4-10. Cortes transversales secuenciales de raíz principal de Pfaffia gnaphalioides (L. Fil.) Mart. 4. A 0,5 cm del ápice. 5 . A 1,5 cm del ápice. 6. A 3,5 cm del ápice. 7. A 3,5 cm del ápice, mostrando el contenido de almidón en el parénquima. 8. A $6 \mathrm{~cm}$ del ápice. 9 . A $22 \mathrm{~cm}$ del ápice. 10. A $50 \mathrm{~cm}$ del ápice. Los cambia adicionales se indican con flechas. $\mathrm{A}=$ almidón; $\mathrm{C}=$ cambium; $\mathrm{CS}=$ corteza secundaria; $\mathrm{F}=$ floema $\mathrm{ML}=$ meristema lateral $\mathrm{Pa}=$ parénquima; $\mathrm{X}=$ xilema. 
cambium adicional ha cesado su actividad y el segundo cambium se encuentra en diferenciación (Fig. 6). A la misma altura del ápice se observa el contenido de almidón en el parénquima (Fig. 7). A los $6,0 \mathrm{~cm}$ se distingue el tercer cambium (Fig. 8), a los $22 \mathrm{~cm}$ el cuarto, ambos con aproximadamente nueve cordones vasculares (Fig. 9), y a los $50 \mathrm{~cm}$ (Fig. 10), el quinto cambium adicional ha comenzado su desarrollo. La estructura anatómica resultante presenta una abundante proporción de parénquima reservante y ausencia de radios parenquimáticos.

Este modelo de crecimiento secundario inusual con sucesivos cambia adicionales originados a partir de un meristema lateral fue descripto en tallos y raíces de algunas especies de Nyctaginaceae por Carlquist (2004). Sin embargo, existe otra postura que sostiene que los cambia adicionales son originados por el parénquima floemático, como fue observado en tallos de distintas especies de Amaranthaceae (Balfour 1965; Rajput \& Rao 1999; Rajput 2001; 2002), Chenopodiaceae (Metcalfe \& Chalk 1957, 1983; Gattuso \& Gattuso 1985) y algunas Nyctaginaceae (Rajput \& Rao 1998).

La ausencia de radios parenquimáticos en Pfaffia gnaphalioides se relaciona con lo expresado por Philipson \& Ward (1965) y Rajput (2001), quienes consideran que este carácter estaría asociado con una reducción de la actividad cambial donde es suprimida en parte la diferenciación de células iniciales radiales, a la vez que indica una tendencia hacia un hábito herbáceo de toda la planta.

Pate (1993) considera que el potencial de reserva de las raíces está determinado principalmente por la proporción de parénquima en contraste con el tejido vascular y otros tejidos no reservantes. El amplio predominio de tejido parenquimático conteniendo almidón en la raíz principal de Pfaffia gnaphalioides (Fig. 7) podría estar facilitando el rápido rebrote en primavera (Baur Höch et al. 1990; Rosnes et al. 1993). Asimismo el desarrollo de una raíz con alta proporción de parénquima y elementos vasculares inmersos en él, concuerda con lo expresado por Esau (1985) quién relaciona la presencia de estructuras inusuales en raíz con acumulación de reservas. Teniendo en cuenta lo expresado por Canny (1998), el almidón favorecería la absorción de agua por parte de la raíz cuando se encuentra disponible en el suelo. Este último concepto sumado a la persistencia de la parte subterránea de Pfaffia gnaphalioides en invierno y al rápido rebrote de la parte aérea en primavera, permitirían la instalación de la especie en el ambiente estudiado.

\section{Referencias bibliográficas}

Balfour, E. 1965. Anomalous secondary thickening in Chenopodiaceae, Nyctaginaceae and Amaranthaceae. Phytomorphology 15: 111-122.

Baur Höch, B.; Mächler, F. \& Nösberger, J. 1990. Effect of carbohydrate demand on the remobilization of starch in stolons and roots of white clover (Trifolium repens L.) after defoliation. Journal of Experimental Botany 41: 573-578.

Canny, M. 1998. Applications of the compensating pressure theory of water transport. American Journal of Botany 85(7): 897.

Carlquist, S. 2004. Lateral meristems, sucessive cambia and their products: a reinterpretation based on roots and stems of Nyctaginaceae. Botanical Journal of Linnean Society 146: 129-143.

Esau, K. 1985. Anatomía Vegetal. Barcelona, Ediciones Omega S.A.

Fahn, A. 1985. Anatomía Vegetal. Madrid, Ediciones Pirámide.

Gattuso, M. \& Gattuso, S. 1985. Estudio del desarrollo del crecimiento inusual en el tallo de Chenopodium ambrosioides L. (Chenopodiaceae). Physis 43: 53-56.

Johansen D.A. 1940. Plant microtechnique. Nueva York, McGraw-Hill Book Co. Inc.

Kagiki, F.; Goncalves, G.; Oliveira, E.; Crocomo, O. \& Gallo L. 2004. Indução de calos e produção de saponinas totais em Pfaffia glomerata (Spreng.) Pedersen in vitro. Revista Brasileira de Plantas Medicinais 7(1): 43-50.

Kutschera-Mitter, L. 1960. Wurzelatlas mitteleuropaischer Ackerunkrauter und Kulturpflanzen. DGL. Frankfurt/ Main.Verlags-GmbH 574 S.

Kutschera, L. \& Lichtenegger E. 1992. Wurzelatlas mitteleuropäischer Grünlandpflanzen. Stuttgart Gustav Fischer Verlag.

Lynch, J. 1995. Root Architecture and Plant Productivity. Plant Physiology 109: 7-13.

Metcalfe, C.R. \& Chalk, L. 1957. Anatomy of the Dicotyledons. Oxford, Clarendon Press.

Metcalfe, C.R. \& Chalk, L. 1983. Anatomy of the Dicotyledons. Oxford, Clarendon Press.

Neto, A.; Costa J.; Belati, C.; Vinholis, A.; Possebom, L.; Da Silva Filho, A.; Cunha, W.; Carvalho, J.; Bastos, J. \& Silva M. 2005. Analgesic and anti-inflamatory activity of a crude root extract of Pfaffia glomerata (Spreng) Pedersen. Journal of Ethnopharmacology 96(1-2): 87-91.

Pate, J.S. 1993. Structural and functional responses to fire and nutrient stress: case studies from the sandplains of south - west Australia. Pp. 189-205. In: L. Fowden; T. Mansfield \& J. toddart (eds.). Plant Adaptation to Environmental Stress. Londres, Chapman \& Hall..

Philipson, W.R. \& Ward, J.M. 1965. The ontogeny of vascular cambium in the stem of seed planta. Biological Review 40: 534-579. 
Rajput, K.S. \& Rao, K.S. 1998. Cambial anatomy and absence of rays in the stem of Boerhaavia species (Nyctaginaceae). Annales Botanici Fennici 35: 131-135.

Rajput, K.S. \& Rao K.S. 1999. Structural and development studies on cambial variant in Pupalia lappacea (Amaranthaceae). Annales Botanici Fennici 36: 137-141.

Rajput, K.S. 2001. Secondary growth of the stem of Celosia argentea L. and Aerva sanguinolenta (L.) Blume (Amaranthaceae). Phyton-Horn 41: 169-177.

Rajput, K.S. 2002. Stem anatomy of Amaranthaceae: Rayless nature of xylem. Flora-Jena 197: 224-232.
Rosnes, K.; Junttila, O.; Ernsten, A. \& Sandli, N. 1993. Development of cold tolerance in white clover (Trifolium repens L.) in relation to carbohydrate and free amino acid content. Acta Agriculturae Scandinavica Section B. Soil and Plant Science 43: 151-155.

Schubert, R. 1991. Root research in natural ecosystem. Pp. 344-349. In: Mc. Michael \& H. Persson (eds.). Plant roots and their environment. Amsterdam, Elsevier Science Publishers.

Vigo, C.; Narita, E.; Milanese-Guterre, M. \& Marques, L. 2004. Caracterizacão farmacognóstica comparativa de Pfaffia glomerata (Spreng.) Pedersen e Hebanthe paniculata Martius - Amaranthaceae. Revista Brasileira de Plantas Medicinais 6(2): 7-19. 